\title{
Concentration-dependent regulation of thyrotropin receptor function by thyroid-stimulating antibody
}

\author{
Takao Ando, Rauf Latif, and Terry F. Davies
}

Division of Endocrinology, Diabetes, and Bone Diseases, Department of Medicine, Mount Sinai School of Medicine, New York, New York, USA.

\begin{abstract}
Thyrotropin receptor (TSHR) Ab's of the stimulating variety are the cause of hyperthyroid Graves disease. MS-1 is a hamster $\mathrm{mAb}$ with TSHR-stimulating activity. To examine the in vivo biological activity of MS-1, mice were treated with purified MS-1 intraperitoneally and the thyroid response evaluated. MS-1 induced a dose-dependent increase in serum thyroxine (T4), with a maximum effect after $10 \mu \mathrm{g}$ of MS-1 was administered. MS-1-secreting hybridoma cells were then transferred into the peritoneum of nude mice to study chronic thyroid stimulation. Serum MS-1 levels detected after 2 weeks were approximately $10-50 \mu \mathrm{g} / \mathrm{ml}$, and the serum TSH was suppressed in all animals. Serum triiodothyronine levels were elevated, but only in animals with low serum MS-1 concentrations. In addition, there was a negative correlation between serum T4 and the serum MS-1 concentrations. These in vivo studies suggested a partial TSHR inactivation induced by excessive stimulation by MS-1. We confirmed this inactivation by demonstrating MS-1 modulation of TSHR function in vitro as evidenced by downregulation and desensitization of the TSHR at concentrations of MS-1 achieved in the in vivo studies. Thus, inactivation of the TSHR by stimulating TSHR autoantibodies (TSHRAb's) in Graves disease patients may provide a functional explanation for the poor correlation between thyroid function and serum TSHR-Ab concentrations.
\end{abstract}

\section{Introduction}

Graves disease is one of the more common human autoimmune diseases (1-3) and is caused by the development of thyrotropin receptor (TSHR) autoantibodies (TSHR-Ab's) that bind to and stimulate the TSHR. The overstimulated thyroid gland produces excessive thyroid hormones in an unregulated manner and induces a hyperthyroid state. However, TSHR-Ab's in the sera of Graves patients have been difficult to study since their discovery $(4)$ and isolation $(5,6)$ because of the presence of multiple antibodies with both TSH-agonist (1-3) and TSH-antagonist (7) activities, as well as their low serum concentration $(8,9)$. To overcome these difficulties, animal models of Graves disease have been developed using immunization with TSHR antigen. We now know that the native conformation of the TSHR is of paramount importance in the induction of stimulating TSHR-Ab's but not of blocking TSHR-Ab's (10). On the basis of successful animal models (11-13), high-affinity mAb's to the TSHR with thyroidstimulating properties have been developed (14-16), including one human TSHR mAb (17). We have been characterizing a highly potent hamster-derived mAb to the TSHR (MS-1) (14) with thyroid-stimulating activity. We obtained this $\mathrm{mAb}$ from a hyperthyroid Armenian hamster immunized with adenovirus vector incorporating the human full-length TSHR (18). We have previously shown that MS-1 was able to stimulate the human TSHR in concentrations as low as $20 \mathrm{ng} / \mathrm{ml}$, and that it recognized a conformational epitope on the $\alpha$ subunit of the TSHR. We have

Nonstandard abbreviations used: $\mathrm{CHO}$ cell lines stably expressing the human thyrotropin receptor (CHO-hTSHR); CHO cell lines stably expressing the mouse thyrotropin receptor (CHO-mTSHR); thyrotropin (TSH); thyrotropin receptor (TSHR); thyrotropin receptor autoantibody (TSHR-Ab); thyroxine (T4); triiodothyronine (T3). Conflict of interest: The authors have declared that no conflict of interest exists.

Citation for this article: J. Clin. Invest. 113:1589-1595 (2004).

doi:10.1172/JCI200421334 also shown that this epitope excludes the cleaved region of the ectodomain (approximately residues 316-366) (14).

Upon binding of TSH or stimulating TSHR-Ab's to the TSHR, signal transduction is initiated via coupling to Gs protein $(1,3)$. Such activation may then be followed by inactivation as a result of G-protein uncoupling (desensitization) and receptor loss from the plasma membrane (downregulation). TSHR desensitization has been extensively studied in thyroid cells following TSH stimulation (19-23). TSHR desensitization, but not downregulation, by TSHR-Ab's from Graves patients' sera has also been documented in vitro (24-26). However, the clinical relevance of such TSHR-Abinduced inactivation in Graves disease is unclear, since the disease results from sustained overstimulation of the TSHR.

To explore further the mechanisms of action of TSHR-Ab's, we have studied the acute and chronic in vivo effects of MS- 1 . These studies showed the potent thyroid-stimulating activity of MS- 1 in vivo but also suggested TSHR inactivation induced by excessive TSHR stimulation. This was confirmed by in vitro studies that showed that the levels of MS-1 achieved in vivo were saturating not only for binding and stimulation, but also for downregulation and desensitization of the TSHR.

\section{Results}

MS-1 stimulates the mouse TSHR in vitro. We first examined the binding and thyroid stimulation of MS- 1 using CHO cell lines stably expressing the mouse thyrotropin receptor (CHO-mTSHR). As shown in Figure 1, MS-1 bound to the mouse TSHR with high affinity $\left(K_{\mathrm{d}}=\sim 1.1 \mathrm{nM}\right)$ and was able to induce cAMP generation with $<10$ $\mathrm{ng} / \mathrm{ml}$; these data are similar to those obtained previously with the human TSHR (14). Thus, MS-1 stimulation and binding of human and mouse TSHRs seemed to be similar.

Acute in vivo thyroid stimulation study. We observed a rapid and robust increase in serum thyroxine (T4) 24 hours after a single 

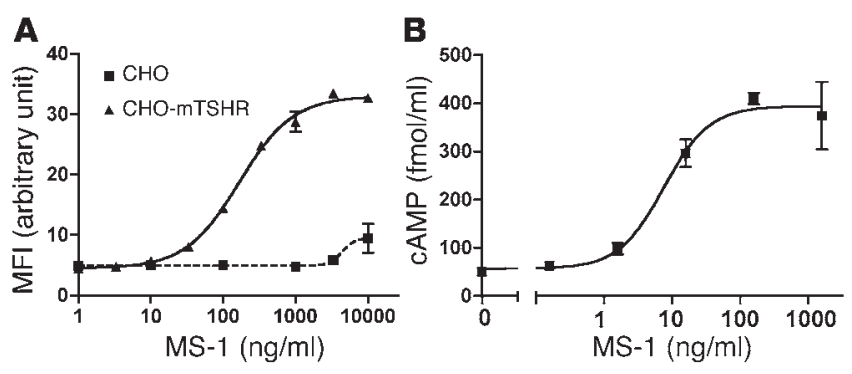

Figure 1

Mouse TSHR binding and stimulation by MS-1 in vitro. (A) Binding of MS-1 on CHO-mTSHR cells (solid line) and nonspecific binding to control $\mathrm{CHO}$ cells (broken line), expressed as mean fluorescence intensity (MFI). (B) CHO-mTSHR cells were stimulated for 2 hours with indicated concentrations of MS-1. Significant stimulation (more than twofold cAMP production compared with basal) was obtained with less than $10 \mathrm{ng} / \mathrm{ml}$ MS-1.

intraperitoneal injection of MS-1. Mice were injected with $0.5,2.5$, 5 , and $10 \mu \mathrm{g}$ of MS-1 $(n=4$ for each), and serum T4 levels measured. The normal range for $\mathrm{T} 4(<5.7 \mu \mathrm{g} / \mathrm{dl})$ was determined by measuring the thyroid hormone levels in the animals before treatment $(n=20)$. A dose-dependent increase in T4 was observed after injection of MS-1, and hyperthyroidism was clearly induced with $5-\mu \mathrm{g}$ and $10-\mu \mathrm{g}$ treatments, while only a marginal increase in T4 was observed with $2.5 \mu \mathrm{g}$ (Figure $2 \mathrm{~A}$ ). There was a robust increase in T4 levels after the $10-\mu \mathrm{g}$ injection, followed by a significant decrease in T4 ( 24 or 48 hours vs. 72 hours; $P<0.01)$. In contrast, a lesser but sustained increase in T4 was observed after the $5-\mu \mathrm{g}$ treatment. When the areas under the curve of $\mathrm{T} 4$ levels were calculated to evaluate the total release of thyroid hormone for each animal (Figure 2B), there was a significantly greater $\mathrm{T} 4$ release with $2.5-\mu \mathrm{g}$ or higher doses of MS- 1 compared with control, and there was a sigmoid relation between the dose of MS-1 injected and the amounts of T4 released $(r=0.9743)$. This indicated that a $10 \mu \mathrm{g}$ dose was near saturating for in vivo thyroid stimulation.

The serum levels of MS-1 (Table 1) indicated that oversaturating concentrations $(>\sim 1 \mu \mathrm{g} / \mathrm{ml})$ failed to sustain the initial high level of thyroid stimulation. Histological examination showed thyroid epithelial cell hypertrophy in the specimens from the $10-\mu \mathrm{g}$ and 5 - $\mu$ g injections of MS- 1 , but not in the lower doses or the control (Figure 2C). Colloid contents were reduced but not totally depleted by the treatment.

Chronic thyroid stimulation study. Having shown potent in vivo bioactivity of MS-1, we studied chronic thyroid stimulation in athymic nude mice $(n=15)$ to avoid any unfavorable immune reactions due to longer exposure of the allogeneic hamster mAb. MS-1-producing hybridoma cells were injected intraperitoneally and the animals examined 2 weeks later. Serum MS-1 levels, 7.2-49.7 $\mu \mathrm{g} /$ $\mathrm{ml}$ (mean $18.5 \pm 3.9 \mu \mathrm{g} / \mathrm{ml}$ ), determined by capture ELISA, were higher than that observed in the acute stimulation study. However, we found that the serum T4 levels of these mice were similar to pretreatment controls $(4.4 \pm 0.3 \mathrm{vs} .5 .3 \pm 0.7 \mu \mathrm{g} / \mathrm{dl})$, and there was even a negative correlation between serum levels of T4 and MS-1 $(r=0.614, P<0.05)$ (Table 2, Figure 3B). It has been shown previously that pristane treatment triggers chronic inflammation (27) and activates intraperitoneal macrophages with increased secretion of cytokines, such as IL- 6 and IL-1 $\beta$ (28). These cytokines have been shown to induce non-thyroid illness syndrome, also called low triiodothyronine/thyroxine (T3/T4) syndrome (29), which might have explained the normal T4 values. However, the serum T3 levels of these mice at the end of the 2 weeks were significantly elevated $(63.53 \pm 4.0$ vs. $43.65 \pm 5.1 \mathrm{ng} / \mathrm{dl}, P<0.03)$ (Figure $3 \mathrm{~A}$, Table 2), and their TSH levels were suppressed or undetectable (Table 2). Therefore, the selective increase in $\mathrm{T} 3$ seen in 7 out of 10 animals examined was proof of hyperthyroidism in the form of $\mathrm{T} 3$ toxicosis, which is sometimes a feature of Graves disease (30, 31). This was further supported by their thyroid histology, which showed epithelial cell hypertrophy in 9 of the 12 animals (Figure 3C) along with colloid depletion (Figure 3D). However, three animals showed evidence of thyroid atrophy (Figure 3E). These data also made it improbable that we were observing direct stimulation of pituitary TSHRs (32) causing the low TSH levels (33).

$M S-1$ biological activity in mouse serum. To explain the negative correlation between serum levels of MS-1 and thyroid hormone, it was important to examine its biological activity in the mice under study. We examined the binding and stimulation of the TSHR by MS- 1 in the mouse serum and found that all of the sera were positive for hamster-derived TSHR-Ab and negative for mouse-derived TSHR-Ab when studied by FACS using CHO-mTSHR cells as targets (Figure 3G). Sera were serially diluted, and the binding to CHO-mTSHR cells was examined using known concentrations of purified MS-1 as control. This allowed an approximate estimation of MS- 1 activity in the sera. These data were comparable to the ELISA-derived concentrations (data not shown) and were further supported by the potent thyroid-stimulating activity of the sera using the cAMP generated by CHO-mTSHR target cells (representative results are shown in Figure $3 \mathrm{H}$ ). We concluded that there was no significant loss of biological activity of MS- 1 and that the

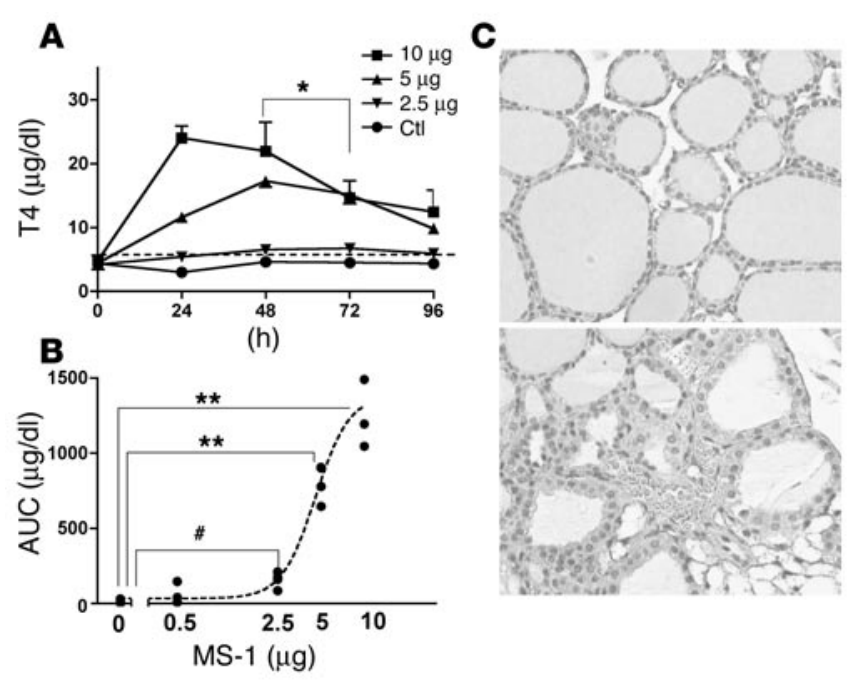

\section{Figure 2}

Acute thyroid stimulation in vivo by MS-1. (A) CBA/J mice were injected with the indicated doses of MS-1 or control (Ctl) Ab, and T4 levels were measured every 24 hours. A broken line indicates the upper level for control serum T4. ${ }^{*} P<0.01$ in T4 levels between 48 hours and 72 hours with a 10- $\mu$ g injection. (B) Area under the curve (AUC) of the T4 released in response to indicated doses of MS-1 stimulation. ${ }^{\#} P<0.002$ and ${ }^{* *} P<0.0001$ in AUC compared with the control treatment. (C) Example of thyroid histology at 96 hours in control (upper) and MS-1 $(10 \mu \mathrm{g})$ treated animals (lower). Note thyroid epithelial cell hypertrophy. Magnification, $\times 400$. 
Table 1

Serum levels of MS-1 in the acute thyroid stimulation study

$\begin{array}{lcc}\text { MS-1 dose } & \text { 24 hours } & \text { 96 hours } \\ 10 \mu \mathrm{g} & 6.40 \pm 2.1 & 4.00 \pm 1.50 \\ 5 \mu \mathrm{g} & 1.80 \pm 1.1 & 0.25 \pm 0.03 \\ 2.5 \mu \mathrm{g} & 0.12 \pm 0.02 & <0.05\end{array}$

The serum levels of hamster $\mathrm{lgG}(\mu \mathrm{g} / \mathrm{ml})$ are shown after the indicated doses of MS-1 treatment as determined by capture ELISA.

thyroid glands must have become refractory to chronic stimulation with oversaturating concentrations of MS-1.

TSHR stimulation in vitro by MS-1. We first examined cAMP production as an indicator of TSHR stimulation induced by various doses of MS-1 for 2, 24, and 48 hours using CHO cell lines stably expressing human TSHR (CHO-hTSHR). TSHR stimulation was sustained for 24 hours as indicated by similar dose-dependent cAMP profiles. However, there was a significant reduction in the cAMP profile after 48 hours of stimulation (Figure 4A). By plotting a ratio for the cAMP produced with each concentration of MS- 1 at 2 hours compared to 48 hours of stimulation (Figure 4B), a dose-dependent loss of TSHR responsiveness was seen in concentrations of more than $300 \mathrm{ng} / \mathrm{ml} \mathrm{MS}-1$ (Figure 4B). This loss of stimulation could be explained by either loss of biological activity of MS-1 or loss of TSHR function.

TSHR binding by MS-1. We first asked whether MS-1 was still bound to the TSHR when the loss of responsiveness was observed. To study TSHR occupancy by MS-1, CHO-hTSHR cells were stimulated for 48 hours and surface MS-1 binding was detected. As shown in Figure 4C, dose-dependent MS-1 binding occurred. However, the maximum MS-1 binding was less than previously observed. Therefore, we suspected that TSHR expression was decreased by MS-1 treatment.

TSHR downregulation. To quantitate the total expression of TSHRs on the plasma membrane in response to MS-1 stimulation, we used our TSHR mAb TAb-8 labeled with green fluorescent dye. TAb-8 can bind to the TSHR without interfering with, or being inhibited by, MS-1 binding to the TSHR (Figure 5A), since it has an independent epitope (Ando et al., unpublished observations). By using labeled TAb-8, we were able to study the total surface TSHRs avoiding a problem with occupancy and, thus, we were able to detect actual TSHR expression. We observed downregulation that was time- and dose-dependent, seen maximally after 48 hours of MS-1 stimulation (Figure 5B). We concluded that loss of surface TSHR was one of the mechanisms of TSHR inactivation induced by MS- 1 .

TSHR desensitization. We also studied the function of TSHRs that had been previously occupied with MS-1. For this purpose, prebound MS-1 was removed from the cell surface TSHRs by washing with cold acid buffer (34). We first optimized the washing procedure by studying MS-1 detaching and binding after an acid wash, and then binding MS-1 to CHO-hTSHR cells after such previous MS-1 detachment. A 30-second exposure of cells to acid buffer was as effective as 4 minutes of exposure and detached about $80 \%$ of bound MS- 1 (Figure 6, A and C). Importantly, the 30-second exposure maintained cell viability (about 95\% vs. $80-85 \%$ ) and MS-1 rebinding (about $50 \%$ vs. about $30 \%$ ) superior to the 4-minute exposure (Figure 6, B and D). Thus, a 30 second acid wash could be used to detach MS-1 bound to CHOhTSHR cells. Although the acid wash procedure attenuated cAMP production by about $40-50 \%$, TSHR desensitization following MS- 1 stimulation was not seen with 4 hours of prior MS- 1 exposure. However, after 24 hours and 48 hours of MS-1 stimulation there was a profound decrease in cAMP generation (Figure 6E). This was more obvious with the addition of isobutylmethylxanthine to the incubations. These results clearly indicated that TSHR function was impaired as a result of desensitization in addition to downregulation induced by MS- 1 , since reduced stimulation was seen even after the removal of MS-1 from the occupied TSHRs. Furthermore, these reduced responses were observed at the same or lesser levels of MS-1 than observed in the in vivo studies.

\section{Discussion}

Since MS-1, a TSHR-stimulating $\mathrm{mAb}$, is of hamster origin, we were able to quantitatively study in vivo thyroid stimulation by a stimulating antibody using mouse hosts. We demonstrated the acute, potent dose-dependent biological activity of MS-1 and found that chronic oversaturating stimulation resulted in the inactivation of TSHRs as suggested by a negative correlation with serum thyroid hormone. The presence of in vivo TSHR inactivation was confirmed in vitro by documenting TSHR desensitization and TSHR downregulation by MS-1 using concentrations of MS-1 similar to those seen in the in vivo studies.

The acute MS-1 stimulation study was similar to the original long-acting thyroid stimulator assays (4), except that we used the direct measurement of thyroid hormone released in response to stimulation. This study clearly showed the potent in vivo biological activity of MS-1 as indicated by a robust increase in serum T4 with acute and oversaturating concentrations of Ab. Similar findings have been obtained with repetitive TSH stimulation (35). As

\section{Table 2}

Thyroid parameters in the chronic thyroid stimulation study

\begin{tabular}{lccccc}
\hline & $\begin{array}{c}\text { Serum MS-1 } \\
(\mu \mathbf{g} / \mathbf{m l})\end{array}$ & $\begin{array}{c}\text { T3 } \\
(\mathbf{n g} / \mathbf{d l})\end{array}$ & $\begin{array}{c}\text { T4 } \\
(\mu \mathbf{g} / \mathbf{m l})\end{array}$ & $\begin{array}{c}\text { TSH } \\
(\mu \mathrm{U} / \mathrm{ml})\end{array}$ & $\begin{array}{c}\text { Thyroid } \\
\text { gland }\end{array}$ \\
C1A & UD & 48.86 & 4.99 & 30 & NA \\
C2 & UD & 33.50 & 5.63 & 105 & NA \\
C3 & UD & 48.60 & 3.39 & 53 & NA \\
C4 & UD & NA & 6.90 & 47 & NA \\
Mean \pm SE & - & $43.65 \pm 5.1$ & $5.3 \pm 0.7$ & $58.3 \pm 16.2$ & - \\
N1 & 42.9 & 48.56 & 2.45 & $<10$ & Atrophy \\
N2 & 11.7 & 53.22 & 3.33 & $<10$ & Hypertrophy \\
N3 & 12.4 & 54.19 & 4.53 & $<10$ & NA \\
N4 & NA & NA & NA & NA & Hypertrophy \\
N5 & 14.7 & 58.69 & 4.5 & 18 & Hypertrophy \\
N6 & 12.1 & 85.08 & 6.35 & $<10$ & Hypertrophy \\
N7 & 17.8 & 63.69 & 3.96 & $<10$ & Atrophy \\
N8 & 20.6 & 69.04 & 3.95 & $<10$ & Hypertrophy \\
N9 & 49.7 & 51.2 & 4.32 & $<10$ & Hypertrophy \\
N10 & 14.3 & 71.12 & 4.02 & $<10$ & Hypertrophy \\
N11 & 7.2 & NA & 6.01 & $<10$ & Hypertrophy \\
N12 & 10 & NA & 5.11 & $<10$ & Hypertrophy \\
N13 & 8.7 & 80.51 & 4.58 & $<10$ & Atrophy \\
Mean \pm SE & $18.5 \pm 3.9$ & $63.53 \pm 4.0$ & $4.4 \pm 0.3$ & - & - \\
& & & & & \\
\hline
\end{tabular}

${ }^{A} \mathrm{C}$ indicates preimmune controls; $\mathrm{N}$ indicates test animals. UD, undetectable; NA, not available. 


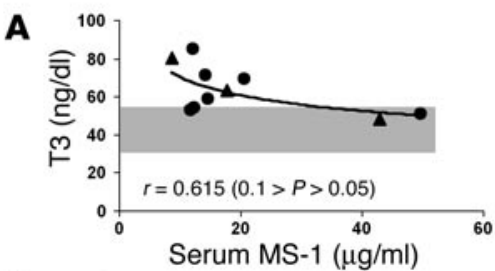

B

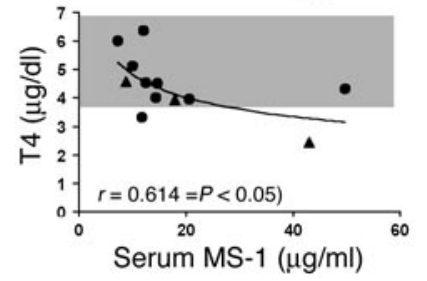

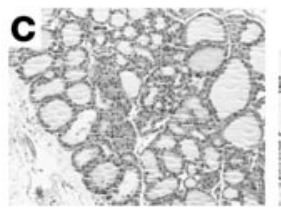
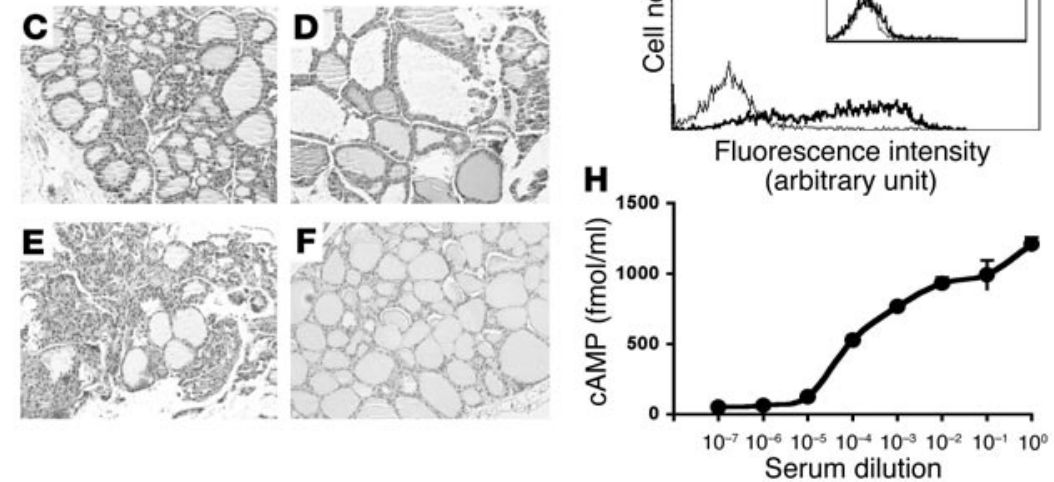

Figure 3

Chronic thyroid stimulation in vivo by MS-1. (A and B) Correlation between serum MS-1 levels and (A) T3 and (B) T4 concentrations. The gray areas indicate normal ranges (mean $\pm 2 \mathrm{SE}$ ). Data for animals with thyroid hypertrophy and atrophy were expressed as filled circles and filled triangles, respectively. (C-F) Thyroid glands from nude mice, showing (C) thyroid epithelial hypertrophy with vascular engorgement (D) thyroid epithelial hypertrophy with colloid depletion (E) thyroid atrophy, and (F) a normal thyroid. Magnification, $\times 200$. (G) Treated mouse serum bound to $\mathrm{CHO}-\mathrm{mTSHR}$ (thick line) and not to control CHO cells (thin line). Anti-hamster IgG and anti-mouse IgG (inset) were used to detect IgG bound to the cells. The horizontal axis indicates fluorescence intensity and the vertical axis the cell number. (H) Representative serum from an MS-1 hybridoma-treated nude mouse containing approximately $50 \mu \mathrm{g} / \mathrm{ml}$ of MS-1 was serially diluted and used for stimulation of CHO-mTSHR cells.

a consequence of secondary TSHR desensitization, however, such active hormone release did not persist (36). It has been shown that only a partial reduction in cAMP production by desensitized TSHRs was sufficient to turn off downstream signaling (36). In contrast to high concentrations of MS-1, we found that the acute treatment with lower levels of $\mathrm{Ab}$ induced a more sustained, but reduced, serum $\mathrm{T} 4$ response in vivo and cAMP response in vitro. Although there have been few studies examining chronic exposure of TSHR-Ab's in vivo, a previous report indicated that Graves patient serum did not induce desensitization of TSHR; however the absence of a robust initial increase in T4 levels suggested that the TSHR-Ab's utilized possessed low potency relative to MS-1 (37). The precise molecular mechanisms preventing thyroid hormone release in conditions of oversaturating stimulation are uncertain, but megalin may have a role in this regulation. Megalin is a lipoprotein expressed on the apical surface of thyrocytes and its expression is upregulated by TSH and stimulating TSHR-
Ab's. Megalin transports thyroglobulin from colloid to the bloodstream, and this transcytosis of thyroglobulin competes with thyroid hormone release from colloid (38).

We have shown that, in vitro, the loss of TSHR function expressed on CHO cells in response to stimulation with MS-1 in concentrations greater than $300 \mathrm{ng} / \mathrm{ml}$ was due to desensitization and downregulation. These data were compatible with previous reports showing TSHR desensitization in vitro by TSHR-Ab's (from Graves disease patients) (24-26) using thyroid cell cultures. However, with TSHR-transfected CHO cells there have been conflicting reports regarding desensitization induced by TSH (39-42), and this most likely depended upon the characteristics of the $\mathrm{CHO}$ cells used (43). As far as we know, there are no reports showing TSHR downregulation induced by TSHR-Ab's in such models. This is most probably due to the low concentration of TSHR-Ab's in sera from Graves disease patients $(8,9)$ and the earlier lack of tools to detect surface TSHRs occupied by TSHR-Ab.
A

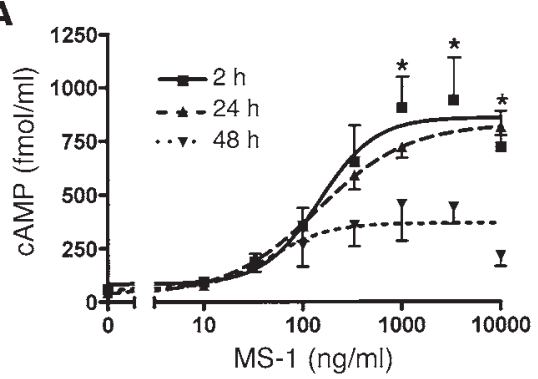

B

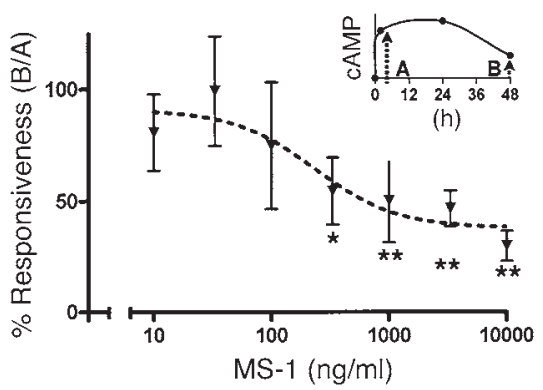

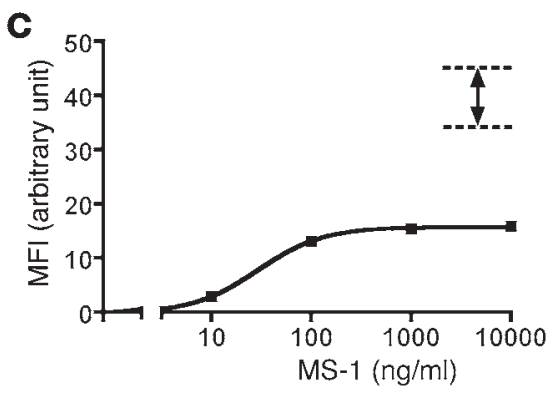

Figure 4

TSHR stimulation study in vitro by MS-1. (A) TSHR activation profile in vitro at different time points. CHO-hTSHR cells were stimulated with indicated doses of MS-1 for indicated periods. ${ }^{*} P<0.05$. (B) TSHR responsiveness with 48 hours of stimulation by MS-1. A ratio for $C A M P$ production with indicated doses of MS-1 (2 hours vs. 48 hours; inset) was expressed as percentage responsiveness. ${ }^{*} P<0.05$; ${ }^{* \star} P<0.02$ (indicated dose vs. 0,10 , and $33 \mathrm{ng} / \mathrm{ml}$ of MS-1). (C) MS-1 binding when loss of TSHR responsiveness was observed. CHO-hTSHR cells were stimulated with the indicated doses of MS-1 for 48 hours, and surface MS-1 binding was studied. MS-1 binding (mean \pm SD) in five separate experiments on $\mathrm{CHO}-\mathrm{hTSHR}$ cells without prior MS-1 stimulation is shown by the arrows. 


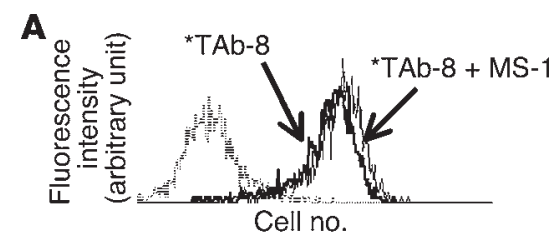

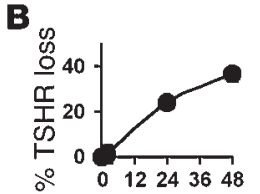

(h)

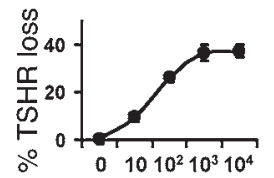

MS-1 (ng/ml)
Figure 5

TSHR downregulation by MS-1. (A) Binding of labeled TAb-8 ( ${ }^{*} \mathrm{TAb}-8$ ) to CHO-hTSHR in the presence (thin line) or absence (thick line) of MS-1. The broken line indicates background staining. (B) TSHR downregulation. Time dependency in response to $1 \mu \mathrm{g} / \mathrm{ml}$ of MS-1 (left) and dose dependency with 48 hours of MS- 1 stimulation (right). Loss of cell surface TSHRs was detected by labeled TAb-8, expressed as percentage TSHR loss after 48 hours. The use of TAb-8 avoided the problem of receptor occupancy by MS-1.

Our studies indicated that optimum levels of stimulating TSHR$\mathrm{Ab}$ are required for in vivo release of $\mathrm{T} 4$ to endure. The optimum level for more sustained thyroid stimulation by MS- 1 was about $100-300 \mathrm{ng} / \mathrm{ml}$. This was near the concentration of mAb necessary for $50 \%$ occupancy of the TSHRs as estimated by receptor-binding studies $\left(K_{\mathrm{d}} \approx 160 \mathrm{ng} / \mathrm{ml}\right)$. The recent isolation of a human TSHRstimulating $\mathrm{mAb}$ has suggested that human TSHR-Ab's may be as potent as or more potent than MS-1 (17). Hence, 'optimum' levels of TSHR-Ab in Graves disease patients may be even lower than $100 \mathrm{ng} / \mathrm{ml}$. Therefore, the low concentration of stimulating TSHR-Ab's in Graves patients' sera may be the reason that hyperthyroidism becomes a chronic problem. It is well known that there is a poor correlation between the concentration of TSHR-Ab and the degree of thyroid hyperfunction in Graves disease (44-48). This has usually been explained by the presence of concomitant TSHR-blocking Ab's (49). However, our study indicated that such a lack of correlation may also be explained by TSHR inactivation induced by high titers of stimulating TSHR-Ab.

In summary, we demonstrated TSHR inactivation in vivo and in vitro by saturating concentrations of the thyroid-stimulating $\mathrm{mAb}$ MS-1. These observations provide an explanation for the clinical significance of TSHR desensitization induced by stimulating TSHR-Ab's and may help explain why low serum levels of TSHR-Ab's in Graves disease patients are able to maintain thyroid hyperfunction.

\section{Methods}

Cells. We used CHO-mTSHR, CHO-hTSHR, and control CHO cells. The cDNA encoding mouse TSHR was provided by P. Kopp (Northwestern University, Chicago, Illinois, USA), and the CHOhTSHR (JPO9) cells (50) were provided by G. Vassart (Université Libre de Bruxelles and Service de Génétique Médicale, Brussels, Belgium). All cells were maintained as previously described (14).

$m A b$ 's. The mAb's MS- 1 and TAb- 8 used in this study were of hamster origin (IgG) against the human TSHR (14). Hybridoma supernatants were purified via Protein G columns (Amersham Biosciences, Piscataway, New Jersey, USA), followed by dialysis against PBS.
Binding-affinity study. CHO-mTSHR cells detached using $1 \mathrm{mM}$ EDTA/EGTA/PBS (Sigma-Aldrich, St. Louis, Missouri, USA) were resuspended at $5 \times 10^{5}$ cells per tube with PBS containing $0.1 \%$ BSA (Sigma-Aldrich) plus $0.01 \%$ sodium azide (Sigma-Aldrich) (FACS buffer), and incubated with the indicated concentrations of hamster $\mathrm{mAb}$. CHO cells were used as a control. Samples were duplicated in all concentrations. After 2 hours of incubation on ice, cells were washed twice with FACS buffer and bound mAb was probed with anti-hamster IgG conjugated with FITC (BD Biosciences Pharmingen, San Diego, California, USA). The mean fluorescence intensity was measured by FACScan (BD Biosciences Pharmingen). Specific binding to the TSHR was obtained by subtracting nonspecific binding to control CHO cells.

TSHR stimulation assay. TSHR stimulation was assessed as previously described (14). In brief, CHO-mTSHR cells seeded at $4 \times 10^{4}$ per well in 96-well plates 1 day prior to the assay were stimulated with MS-1 or mouse serum diluted with fresh medium in the absence of isobutylmethylxanthine to better study physiological stimulation. The intracellular cAMP concentration (in femtomoles per milliliter) was measured by cAMP Direct Biotrak EIA (Amersham Biosciences). Stimulation was expressed as the percentage increase in cAMP based on basal cAMP production. More than $200 \%$ stimulation was taken as positive.

Acute thyroid stimulation study. Female CBA/J mice (6-8 weeks old) were injected intraperitoneally with the indicated doses of purified hamster mAb MS- 1 or control hamster mAb to $\mathrm{CHO}$ cell membrane. Blood was collected every 24 hours until 96 hours after administration to study the levels of mouse serum T4 and hamster Ab. All animals were sacrificed at 96 hours. The thyroid glands of sacrificed animals were fixed in 10\% formalin/PBS and

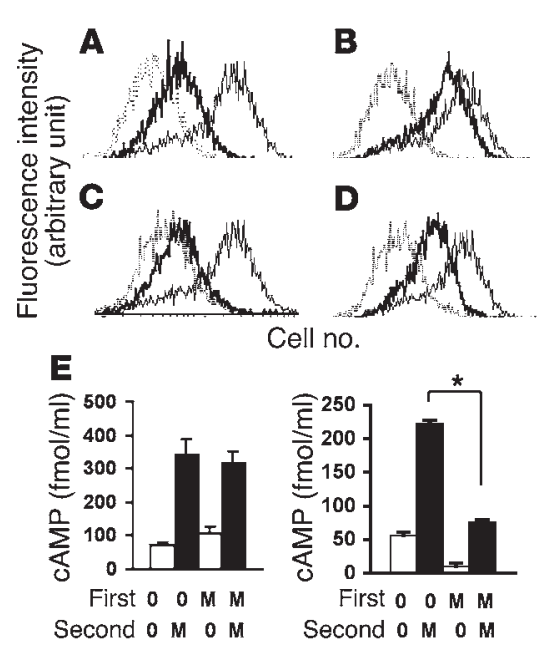

\section{Figure 6}

TSHR desensitization by MS-1. (A-D) Optimization of acid wash. MS-1 binding to CHO-hTSHR cells (thin solid lines), bound MS-1 (thick line) after acid wash for 30 seconds (A) or 4 minutes (C), and rebinding of MS-1 (thick lines) after acid wash for 30 seconds (B) or 4 minutes (D) are shown. Broken lines indicate background staining. (E) TSHR desensitization. CHO-hTSHR cells were stimulated with $1 \mu \mathrm{g} / \mathrm{ml}$ of MS-1 or medium (indicated as M and 0, respectively) for 4 hours (left) and 48 hours (right) (first stimulation), followed by 2 hours incubation with or without the same dose of MS-1 with $2 \mathrm{mM}$ isobutylmethylxanthine (second stimulation). Between these two stimuli, cells were washed with cold acid buffer. Forskolin $(50 \mu \mathrm{M})$ stimulation generated approximately $3,000 \mathrm{fmol}$ of cAMP. ${ }^{*} P<0.001$. 
stained with H\&E. Mice were studied in accordance with protocols approved by the Internal Review Board of Mount Sinai.

Chronic thyroid stimulation study. Pristane $(0.5 \mathrm{ml} / \mathrm{animal})$ (SigmaAldrich) was injected intraperitoneally into female athymic nude mice (Hsd; Harlan Bioproducts for Science Inc., Indianapolis, Indiana, USA; $n=15) 2$ weeks prior to injection of MS- 1 hybridoma cells $\left(2.5 \times 10^{6}\right.$ cells per animal). Two weeks after transferring hybridoma cells, the animals were sacrificed and sera and thyroid glands collected. Pretreatment mouse sera were used as negative controls.

Thyroid hormone and TSH assays. Serum total T3 and T4 concentrations were measured as previously described by a clinical automatic coated-tube assay (51). Mouse serum TSH concentrations were determined by specific RIA using mouse standards as described previously (52) by R. Weiss at the University of Chicago (Chicago, Illinois, USA).

Hamster IgG capture ELISA. The concentration of MS-1 hamster $\mathrm{IgG}$ in mouse serum was determined by an IgG capture ELISA. In brief, mouse serum (1:50 dilution) was added in duplicate to antihamster IgG $(2 \mu \mathrm{g} / \mathrm{ml})$ (Jackson ImmunoResearch Laboratories Inc., West Grove, Pennsylvania, USA) coated microtiter plates. Captured hamster IgG was probed with anti-hamster IgG conjugated with HRP (BD Biosciences Pharmingen) (1:2000). A known concentration of purified MS-1 was used to generate a standard curve from 1 $\mathrm{ng} / \mathrm{ml}$ to $1 \mu \mathrm{g} / \mathrm{ml}$. The sensitivity of this assay was about $1 \mathrm{ng} / \mathrm{ml}$.

Detection of TSHR-binding Ab in mouse serum. Mouse serum was diluted 1:100 with FACS buffer and incubated with either CHO-mTSHR or $\mathrm{CHO}$ cells as described earlier. Anti-hamster IgG conjugated with biotin and streptavidin-conjugated phycoerythrin (1:100) (SigmaAldrich) or anti-mouse IgG conjugated with FITC (Sigma-Aldrich) were used in separate tubes. These secondary Ab's were highly specific, and there was minimal cross reactivity (data not shown).

TSHR stimulation study in vitro by MS-1. CHO-hTSHR cells were seeded at $2 \times 10^{4}$ cells per well in a 96-well plate 1 day prior to stimulation with the indicated doses of MS- 1 in fresh medium for the indicated periods of time without isobutylmethylxanthine, and the intracellular cAMP was measured as described.

TSHR downregulation study. To study TSHR loss in response to MS- 1 stimulation, CHO-hTSHR cells were seeded at $4 \times 10^{5}$ cells per well in a six-well plate 1 day prior to stimulation with MS- 1 for 48 hours. Cells were detached, and bound MS-1 was studied with anti-hamster IgG conjugated with FITC and, in separate tubes, total surface TSHR was studied using TAb-8 labeled with Alexa 488 (Molecular Probes Inc., Eugene, Oregon, USA). TAb-8 binds to the TSHR even when occupied by MS-1.

Optimization of acid wash. To study TSHR desensitization, cold acid buffer (34) was used to unbind MS-1 on CHO-hTSHR cells, to minimize receptor occupancy. CHO-hTSHR cells to which MS$1 \mathrm{mAb}$ 's had been previously bound were washed twice with PBS, incubated with cold acid buffer for the indicated periods, and rinsed once with cold acid buffer followed by washing twice with PBS. Then, cells were detached using EGTA/EDTA and stained with FITC-conjugated anti-hamster IgG to detect residual MS-1, or again stained with MS-1 to study repeat binding. Similarly, $\mathrm{CHO}-$ hTSHR cells, without MS-1 pretreatment, were used to study binding of MS-1 after an acid wash. To examine cell viability, cells were also stained with 7-amino actinomycine (BD Biosciences Pharmingen). Fluorescence intensity was then studied by FACS.

In vitro desensitization studies. CHO-hTSHR cells were seeded at $2 \times 10^{4}$ cells per well in a 96-well plate 1 day prior to stimulation with the indicated doses of MS- 1 in fresh medium for the indicated periods of time. After stimulation, the medium was aspirated and the cells were washed with cold acid buffer for 30 seconds to detach bound MS-1. After rinsing the cells once with cold acid buffer and twice with PBS, cells were restimulated for 2 hours with 1 $\mu \mathrm{g} / \mathrm{ml}$ of MS- 1 in fresh medium with or without $2 \mathrm{mM}$ isobutylmethylxanthine (Sigma-Aldrich). Intracellular cAMP generation was then measured.

Statistical analyses. Data were analyzed using Prism4 (GraphPad Software for Science Inc., San Diego, California, USA) and expressed as mean \pm SE. The Student's $t$ test was used for statistical analysis. $P<0.05$ was considered significant.

\section{Acknowledgments}

We thank Thomas Moran and Alla Pritsker from the Hybridoma Shared Facility, Mount Sinai School of Medicine, for help with the hybridoma production; Pamela Unger from the Department of Pathology for thyroid histology; and Edward Diamond from the Department of Medicine, Mount Sinai Medical Center, for thyroid hormone measurements. We also thank Naoko Arata, Michael Goldberg, Samira Daniel, and Reigh-Yi Lin for helpful discussion. This work was supported in part by NIH grants DK52464, DK45011, and AI24671 to T.F. Davies, and the David Owen Segal Endowment to T. Ando.

Received for publication February 13, 2004, and accepted in revised form March 23, 2004.

Address correspondence to: Takao Ando, Mount Sinai School of Medicine, Box 1055, 1 Gustave L. Levy Place, New York, New York 10029, USA. Phone: (212) 241-4129; Fax: (212) 241-4218; E-mail: takao.ando@mssm.edu.

\footnotetext{
1. Davies, T., Marians, R., and Latif, R. 2002. The TSH receptor reveals itself. J. Clin. Invest. 110:161-164. doi:10.1172/JCI200216234.

2. Rees Smith, B., McLachlan, S.M., and Furmaniak, J. 1988. Autoantibodies to the thyrotropin receptor. Endocr. Rev. 9:106-121.

3. Rapoport, B., Chazenbalk, G.D., Jaume, J.C., and McLachlan, S.M. 1998. The thyrotropin (TSH) receptor: interaction with TSH and autoantibodies. Endocr. Rev. 19:673-716.

4. Adams, D.D., and Purves, H.D. 1956. Abnormal responses in the assay of thyrotropins. Proc. Univ. Otago Med. Sch. 34:11-12.

5. Kriss, J., Pleshakov, V., and Chien, J.R. 1964. Isolation and identification of the long acting thyroid stimulator and its relation to hyperthyroidism and circumscribed pretibial myxedema. J. Clin. Endocrinol. Metab. 24:1005-1028.
}

6. Meek, J.C., Jones, A.E., Lewis, U.J., and Vanderlaan, W.P. 1964. Characterization of the long-acting thyroid stimulator of Graves' disease. Proc. Natl. Acad. Sci. U. S. A. 52:342-349.

7. Matsuura, N., Yamada, Y., and Nohata, Y. 1980. Familial neonatal hypothyroidism due to maternal TSH-binding inhibiting immunoglobulins. N. Engl. J. Med. 303:738-741.

8. Chazenbalk, G.D., Jaume, J.C., McLachlan, S.M., and Rapoport, B. 1997. Engineering the human thyrotropin receptor ectodomain from a non-secreted form to a secreted, highly immunoreactive glycoprotein that neutralizes autoantibodies in Graves' patients' sera. J. Biol. Chem. 272:18959-18965.

9. Jaume, J.C., Kakinuma, A., Chazenbalk, G.D., Rapoport, B., and McLachlan, S.M. 1997. Thyrotropin receptor autoantibodies in serum are pres- ent at much lower levels than thyroid peroxidase autoantibodies: analysis by flow cytometry. J. Clin. Endocrinol. Metab. 82:500-507.

10. Davies, T.F., Vlase, H., and Kita, M. 1999. The search for an animal model for Graves' disease. In The decade of autoimmunity. Y. Shoenfeld, editor. Elsevier Science. Amsterdam, The Netherlands. $43-50$.

11. Shimojo, N., et al. 1996. Induction of Graves-like disease in mice by immunization with fibroblasts transfected with the thyrotropin receptor and a class II molecule. Proc. Natl. Acad. Sci. U. S. A. 93:11074-11079.

12. Costagliola, S., et al. 2000. Genetic immunization of outbred mice with thyrotropin receptor cDNA provides a model of Graves' disease. J. Clin. Invest. 105:803-811.

13. Nagayama, Y., et al. 2002. A novel murine model 
of Graves' hyperthyroidism with intramuscular injection of adenovirus expressing the thyrotropin receptor. J. Immunol. 168:2789-2794.

14. Ando, T., et al. 2002. A monoclonal thyroid-stimulating antibody. J. Clin. Invest. 110:1667-1674. doi:10.1172/JCI200216991.

15. Costagliola, S., et al. 2002. Generation of a mouse monoclonal TSH receptor antibody with stimulating activity. Biochem. Biophys. Res. Commun. 299:891-896.

16. Sanders, J., et al. 2002. Thyroid-stimulating monoclonal antibodies. Thyroid. 12:1043-1050.

17. Sanders, J., et al. 2003. Human monoclonal thyroid stimulating autoantibody. Lancet. 362:126-128.

18. Ando, T., Imaizumi, M., Graves, P., Unger, P., and Davies, T.F. 2003. Induction of thyroid-stimulating hormone receptor autoimmunity in hamsters. Endocrinology. 144:671-680.

19. Nagayama, Y., et al. 1996. Involvement of G protein-coupled receptor kinase 5 in homologous desensitization of the thyrotropin receptor. J. Biol. Chem. 271:10143-10148.

20. Iacovelli, L., Franchetti, R., Grisolia, D., and De Blasi, A. 1999. Selective regulation of $G$ proteincoupled receptor-mediated signaling by G protein-coupled receptor kinase 2 in FRTL-5 cells: analysis of thyrotropin, alpha(1B)-adrenergic, and $\mathrm{A}(1)$ adenosine receptor-mediated responses. $\mathrm{Mol}$. Pharmacol. 56:316-324.

21. Iacovelli, L., Franchetti, R., Masini, M., and De Blasi, A. 1996. GRK2 and beta-arrestin 1 as negative regulators of thyrotropin receptor-stimulated response. Mol. Endocrinol. 10:1138-1146.

22. Lalli, E., and Sassone-Corsi, P. 1995. Thyroid-stimulating hormone (TSH)-directed induction of the CREM gene in the thyroid gland participates in the long-term desensitization of the TSH receptor. Proc. Natl. Acad. Sci. U. S. A. 92:9633-9637.

23. Hirayu, H., Magnusson, R.P., and Rapoport, B. 1985. Studies on the mechanism of desensitization of the cyclic AMP response to TSH stimulation in a cloned rat thyroid cell line. Mol. Cell. Endocrinol. 42:21-27.

24. Vitti, P., et al. 1986. Thyroid-stimulating antibody mimics thyrotropin in its ability to desensitize the adenosine 3', 5'-monophosphate response to acute stimulation in continuously cultured rat thyroid cells (FRT-L5). J. Clin. Endocrinol. Metab. 63:454-458.

25. Damante, G., Foti, D., Catalfamo, R., and Filetti, S. 1987. Desensitization of the thyroid cyclic AMP response to thyroid stimulating immunoglobulin: comparison with TSH. Metabolism. 36:768-773.

26. Madec, A.M., Clavel, S., Stefanutti, A., and Orgiazzi, J. 1988. Blocking anti-thyrotropin receptor antibodies desensitize cultured human thyroid cells. Endocrinology. 123:2062-2066.

27. Potter, M., Wax, J., and Jones, G.M. 1997. Indo- methacin is a potent inhibitor of pristane and plastic disc induced plasmacytomagenesis in a hypersusceptible Balb/c congenic strain. Blood. 90:260-269.

28. Naim, J.O., et al. 2000. Induction of hypergammaglobulinemia and macrophage activation by silicon gels and oils in female A.SW mice. Clin. Diagn. Lab. Immunol. 7:366-370.

29. De Groot, L.J. 1999. Dangerous dogmas in medicine: the nonthyroidal illness syndrome. J. Clin. Endocrinol. Metab. 84:151-164.

30. Hollander, C.S., et al. 1972. Clinical and laboratory observations in cases of triiodothyronine toxicosis confirmed by radioimmunoassay. Lancet. 1:609-611.

31. Shalet, S.M., Beardwell, C.G., Lamb, A.M., and Gowland, E. 1975. Value of routine serum-triiodothyronine estimation in diagnosis of thyrotoxicosis. Lancet. 2:1008-1010.

32. Prummel, M.F., et al. 2000. Expression of the thyroid-stimulating hormone receptor in the folliculo-stellate cells of the human anterior pituitary. J. Clin. Endocrinol. Metab. 85:4347-4353.

33. Brokken, L.J.S., Scheenhart, J.W.C., Wiersinga, W.M., and Prummel, M.F. 2001. Suppression of serum TSH by Graves' Ig: evidence for a functional pituitary TSH receptor. J. Clin. Endocrinol. Metab. 86:4814-4817.

34. Ascoli, M. 1982. Internalization and degradation of receptor-bound human choriogonadotropin in Leydig tumor cells. Fate of the hormone subunits. J. Biol. Chem. 257:13306-13311.

35. Gerber, H., Studer, H., Conti, A., Engler, H., and Kohler, H. 1981. Reaccumulation of thyroglobulin and colloid in rat and mouse thyroid follicles during intense thyrotropin stimulation. J. Clin. Invest. 68:1338-1347.

36. Nielsen, T.B., Totsuka, Y., and Field, J.B. 1982. Three types of desensitization of metabolic responses to thyrotropin in thyroid tissue: a review. Endocrinol. Exp. 16:247-257.

37. Ikeda, H., and Nagataki, S. 1983. Lack of refractoriness to stimulation with long acting thyroid stimulator of thyroid hormone synthesis and thyroid hormone secretion in mice in vivo. Acta Endocrinol. 102:392-395.

38. Marino, M., Pinchera, A., McCluskey, R.T., and Chiovato, L. 2001. Megalin in thyroid physiology and pathology. Thyroid. 11:47-56.

39. Haraguchi, K., et al. 1993. Thyrotrophin-dependent desensitization by Chinese hamster ovary cells that express the recombinant human thyrotrophin receptor. J. Endocrinol. 139:425-429.

40. Tezelman, S., et al. 1994. Desensitization of adenylate cyclase in Chinese hamster ovary cells transfected with human thyroid-stimulating hormone receptor. Endocrinology. 134:1561-1569.
41. Chazenbalk, G.D., Nagayama, Y., Kaufman, K.D., and Rapoport, B. 1990. The functional expression of recombinant human thyrotropin receptors in nonthyroidal eukaryotic cells provides evidence that homologous desensitization to thyrotropin stimulation requires a cell-specific factor. Endocrinology. 127:1240-1244.

42. Heldin, N.E., Gustavsson, B., Hermansson, A., and Westermark, B. 1994. Thyrotropin (TSH)-induced receptor internalization in nonthyroidal cells transfected with a human TSH-receptor complementary deoxyribonucleic acid. Endocrinology. 134:2032-2036.

43. Costagliola, S., et al. 1992. Binding assay for thyrotropin receptor autoantibodies using the recombinant receptor protein. J. Clin. Endocrinol. Metab. 75:1540-1544.

44. Endo, K., et al. 1978. Detection and properties of TSH-binding inhibitor immunoglobulins in patients with Graves' disease and Hashimoto's thyroiditis. J. Clin. Endocrinol. Metab. 46:734-739.

45. Kuzuya, N., et al. 1979. Correlation between thyroid stimulators and 3,5,3'-triiodothyronine suppressibility in patients during treatment for hyperthyroidism with thionamide drug: comparison of assays by thyroid-stimulating and thyrotropin-displacing activities. J. Clin. Endocrinol. Metab. 48:706-711.

46. Docter, R., Bos, G., Visser, T.J., and Hennemann, G. 1980. Thyrotropin binding inhibiting immunoglobulins in Graves' disease before, during and after antithyroid therapy, and its relation to longacting thyroid stimulator. Clin. Endocrinol. (Oxf.) 12:143-153.

47. Adams, D.D., Kennedy, T.H., and Stewart, R.D.H. 1974. Correlation between long-acting thyroid stimulator protector level and thyroid ${ }^{131}$ I uptake in thyrotoxicosis. Br. Med. J. 2:199-201.

48. Mukhtar, E.D., Smith, B.R., Pyle, G.A., Hall, R., and Vice, P. 1975. Relation of thyroid-stimulating immunoglobulins to thyroid function and effects of surgery, radioiodine, and antithyroid drugs. Lancet. 1:713-715.

49. Wilkin, T.J. 1990. Receptor autoimmunity in endocrine disorders. N. Engl. J. Med. 323:1318-1324.

50. Perret, J., et al. 1990. Stable expression of the human TSH receptor in CHO cells and characterization of differentially expressing clones. Biochem. Biophys. Res. Commun. 171:1044-1050.

51. Marians, R.C., et al. 2002. Defining thyrotropindependent and -independent steps of thyroid hormone synthesis by using thyrotropin receptor-null mice. Proc. Natl. Acad. Sci. U. S. A. 99:15776-15781.

52. Pohlenz, J., et al. 1999. Improved radioimmunoassay for measurement of mouse thyrotropin in serum: strain differences in thyrotropin concentration and thyrotroph sensitivity to thyroid hormone. Thyroid. 9:1265-1271. 